\begin{tabular}{|c|c|}
\hline Title & Introduction of the vector potential to a linear MHD simulation code based on a real coordinate system \\
\hline Author(s) & Takado, W.; Matsumoto, Y.; W atanabe, K. Y.; Tomioka, S.; Oikawa, S. \\
\hline Citation & $\begin{array}{l}\text { Physics of plasmas, } 23(10), 102509 \\
\text { https://doi.org/10.1063/1.4965232 }\end{array}$ \\
\hline Issue Date & $2016-10$ \\
\hline Doc URL & http:/hdl.handle.net/2115/67212 \\
\hline Rights & $\begin{array}{l}\text { Copyright } 2016 \text { A merican Institute of Physics. This article may be downloaded for personal use only. A ny other use } \\
\text { requires prior permission of the author and AIP Publishing. The following article appeared in (citation of published } \\
\text { article) and may be found at http://aip.scitation.org/doi/abs/10.1063/1.4965232?journal Code=php. }\end{array}$ \\
\hline Type & article \\
\hline File Information & Introduction_of_the_vector_potential_to_a_linear_M.pdf \\
\hline
\end{tabular}

Instructions for use 


\section{Introduction of the vector potential to a linear MHD simulation code based on a real coordinate system}

Article in Physics of Plasmas · October 2016

DOI: $10.1063 / 1.4965232$

CITATIONS

0

5 authors, including:

Yutaka Matsumoto

Hokkaido University

31 PUBLICATIONS 212 CITATIONS

SEE PROFILE

\section{Shun-Ichi Oikawa}

Hokkaido University

52 PUBLICATIONS 95 CITATIONS

SEE PROFILE
READS

16

Satoshi Tomioka

Hokkaido University

40 PUBLICATIONS 154 CITATIONS

SEE PROFILE 


\section{AIP $\mid$ Physics of \\ Plasmas}

Introduction of the vector potential to a linear MHD simulation code based on a real coordinate system

W. Takado, Y. Matsumoto, K. Y. Watanabe, S. Tomioka, and S. Oikawa

Citation: Physics of Plasmas 23, 102509 (2016); doi: 10.1063/1.4965232

View online: http://dx.doi.org/10.1063/1.4965232

View Table of Contents: http://scitation.aip.org/content/aip/journal/pop/23/10?ver=pdfcov

Published by the AIP Publishing

Articles you may be interested in

Alfvén ionization in an MHD-gas interactions code

Phys. Plasmas 23, 072117 (2016); 10.1063/1.4956443

Numerical linearized MHD model of flapping oscillations

Phys. Plasmas 23, 062905 (2016); 10.1063/1.4954388

Three-dimensional MHD simulation of the dynamic heliosphere

AIP Conf. Proc. 932, 153 (2007); 10.1063/1.2778958

Classical MHD shocks: theory and numerical simulation

AIP Conf. Proc. 781, 42 (2005); 10.1063/1.2032673

3-D Simulations of MHD Jets - The Stability Problem

AIP Conf. Proc. 703, 308 (2004); 10.1063/1.1718472

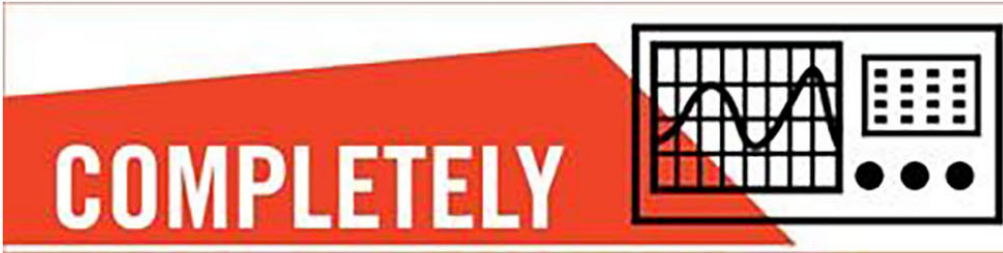

PHYSICS 


\title{
Introduction of the vector potential to a linear MHD simulation code based on a real coordinate system
}

\author{
W. Takado, ${ }^{1}$ Y. Matsumoto, ${ }^{1}$ K. Y. Watanabe,${ }^{2}$ S. Tomioka, ${ }^{1}$ and S. Oikawa ${ }^{1}$ \\ ${ }^{1}$ Hokkaido University, Sapporo 060-8628, Japan \\ ${ }^{2}$ National Institute for Fusion Science, Toki 509-5292, Japan
}

(Received 25 July 2016; accepted 5 October 2016; published online 18 October 2016)

Here, the vector potential was introduced to a linear magnetohydrodynamics (MHD) simulation code, and the modified and original simulation results were compared. The effects of the broken solenoidal condition on the perturbed magnetic field in linear MHD simulations based on real coordinates were investigated. The results showed that the modified code can successfully remove the error in this condition, and the modified code was confirmed to work appropriately. Incorrect results can be yielded by an error in the condition, especially in analyses of the mode structure.

Published by AIP Publishing. [http://dx.doi.org/10.1063/1.4965232]

\section{INTRODUCTION}

The solenoidal condition of the magnetic field $\nabla \cdot \boldsymbol{B}=0$ is included in magnetohydrodynamic (MHD) equations. This condition is not automatically maintained in full MHD simulations because of numerical errors. Brackbill and Barnes studied the effect of nonzero $\nabla \cdot \boldsymbol{B}$ on the MHD simulations and found that the nonphysical force parallel to the magnetic field is generated by nonzero $\nabla \cdot \boldsymbol{B}$ errors. ${ }^{1}$ They found that the energy conservation law can be satisfied by writing the momentum equation in the nonconservation form even when $\nabla \cdot \boldsymbol{B} \neq 0$. Even if this method is applied, however, a concern is that the nonphysical force term cannot be eliminated and will still yield inaccurate results. There have been almost no investigations on how harmful nonzero $\nabla \cdot \boldsymbol{B}$ errors are in full MHD simulations of a fusion reactor.

The Large Helical Device (LHD) is a heliotron-type device with a set of $\ell=2$ (poloidal period number) and $m=10$ (toroidal period number) continuous helical coils. ${ }^{2}$ Recent experimental studies have investigated the effect of independent low-order magnetic fluctuations in high-beta LHD plasmas. ${ }^{3}$ This effect is important for clarifying the influence of the fine flattening structures in the temperature profiles on the corresponding resonant magnetic surfaces. In numerical analyses, low-order fluctuations can easily be obtained if a numerical code based on magnetic coordinates is employed. However, it is necessary to use a numerical code based on real coordinates in order to investigate the properties of the resistive interchange mode in the stochastic region. The initial perturbation is generally given as a random profile in the numerical code based on the real coordinates. Then, high-order fluctuations become more dominant than low-order ones. In order to resolve this problem, a method for investigating the specified single-mode instability has been developed. ${ }^{4}$ However, a large $\nabla \cdot \boldsymbol{B}$ error is expected to yield instabilities with an undesirable mode and disturb the analysis of the specified single-mode instability.

In order to verify this prediction, the vector potential is introduced to a linear MHD simulation code. In this study, the linear million instructions per second (MIPS) $\operatorname{code}^{5}$ was employed. MIPS is a full MHD code that uses real coordinates and has been used for some analyses lately. The linear MIPS code has also been used for the above analysis on a specified single-mode instability. Introducing the vector potential $\boldsymbol{A}$ is a useful way to guarantee $\nabla \cdot \boldsymbol{B}=0$ because the magnetic field is always calculated by the rotation of the vector potential as $\boldsymbol{B}=\nabla \times \boldsymbol{A}$. Although there is some concern about the accuracy for the second derivative of the vector potential, the order of the accuracy remains the same as that of the vector potential if there is no discontinuity in the magnetic field. ${ }^{6}$ In this study, the linear MIPS code was modified by introducing the vector potential. Only the perturbed $\nabla \cdot \boldsymbol{B}$ was considered because it is difficult to eliminate the $\nabla \cdot \boldsymbol{B}$ error from the equilibrium magnetic field. The modified and original linear MIPS simulation results were compared in order to confirm that the modified code works appropriately and can successfully remove the perturbed $\nabla \cdot \boldsymbol{B}$ error. The effect of introducing the vector potential was also investigated.

This paper is organized as follows. Section II provides the numerical model. Section III compares the modified and original linear MIPS simulation results. Finally, the summary and discussion are given in Section IV.

\section{NUMERICAL MODEL}

The original linear MIPS code ${ }^{5}$ solves the following linear MHD equations for the cylindrical coordinates $(R, \phi, Z)$ :

$$
\begin{aligned}
& \rho \frac{\partial \boldsymbol{V}}{\partial t}=-\nabla P_{1}+\boldsymbol{J}_{1} \times \boldsymbol{B}_{0}+\boldsymbol{J}_{0} \times \boldsymbol{B}_{1} \\
&+ \frac{4}{3} \nu \rho \nabla(\nabla \cdot \boldsymbol{V})-\nu \rho \nabla \times \boldsymbol{\omega}, \\
& \frac{\partial \boldsymbol{B}_{1}}{\partial t}=-\nabla \times \boldsymbol{E}_{1}, \\
& \frac{\partial P_{1}}{\partial t}=- \nabla \cdot\left(P_{0} \boldsymbol{V}\right)-(\gamma-1) P_{0} \nabla \cdot \boldsymbol{V} \\
&+(\gamma-1) \eta \boldsymbol{J}_{0} \cdot \boldsymbol{J}_{1}+\chi \nabla^{2} P_{1}, \\
& \boldsymbol{E}_{1}=-\boldsymbol{V} \times \boldsymbol{B}_{0}+\eta \boldsymbol{J}_{1},
\end{aligned}
$$




$$
\begin{gathered}
\boldsymbol{J}_{1}=\frac{1}{\mu_{0}} \nabla \times \boldsymbol{B}_{1}, \\
\boldsymbol{\omega}=\nabla \times \boldsymbol{V} .
\end{gathered}
$$

Here, $\boldsymbol{V}$ is the velocity, $\boldsymbol{B}$ is the magnetic field, $P$ is the pressure, $\boldsymbol{E}$ is the electric field, $\boldsymbol{J}$ is the current density, and $\boldsymbol{\omega}$ is the vorticity. $\rho$ is the density, $\eta$ is the resistivity, $\nu$ is the viscosity, $\chi$ is the thermal diffusion coefficient, $\mu_{0}$ is the vacuum magnetic permeability, and $\gamma$ is the adiabatic constant. The subscripts 0 and 1 indicate the equilibrium and perturbation quantities, respectively (except for $\mu_{0}$ ).

In this code, the fourth-order finite difference scheme is used for the spatial derivatives. The fourth-order RungeKutta method is used for the time integrations. The fixed boundary is assumed to be at the plasma-vacuum boundary.

In the modified linear MIPS code, the vector potential $\boldsymbol{A}$ is introduced to Eq. (2) as follows:

$$
\begin{gathered}
\frac{\partial \boldsymbol{A}_{1}}{\partial t}=-\boldsymbol{E}_{1}-\nabla \varphi_{1}, \\
\boldsymbol{B}_{1}=\nabla \times \boldsymbol{A}_{1},
\end{gathered}
$$

where $\varphi$ is the scalar potential. Here, the treatment of $\varphi_{1}$ is considered. The time derivative of $\boldsymbol{B}_{1}$ can be written by using Eqs. (7) and (8) as follows:

$$
\frac{\partial \boldsymbol{B}_{1}}{\partial t}=\frac{\partial \nabla \times \boldsymbol{A}_{1}}{\partial t}=-\nabla \times \boldsymbol{E}_{1}-\nabla \times \nabla \varphi_{1}=-\nabla \times \boldsymbol{E}_{1} .
$$

Therefore, $\varphi_{1}$ cannot affect the MHD simulation results no matter its value. Thus, the uniform scalar potential $\varphi_{1}=0$ is given and is assumed to be a constant.

The initial equilibrium of the low-beta LHD plasmas with the volume-averaged beta value $\langle\beta\rangle=1.4 \%$ was constructed by using the HINT code ${ }^{7,8}$ for a vacuum magnetic field of $R_{\mathrm{ax}}^{\mathrm{v}}=3.6 \mathrm{~m}, B_{\mathrm{ax}}^{\mathrm{v}}=3 \mathrm{~T}$, and $\gamma_{\mathrm{c}}=1.1967$. Here, $R_{\mathrm{ax}}^{\mathrm{v}}$ is the major radius of the magnetic axis, $B_{\mathrm{ax}}^{\mathrm{v}}$ is the magnetic field strength on the magnetic axis, and $\gamma_{c}$ is the coil pitch parameter. The obtained equilibrium pressure profile on the vertically elongated poloidal plane of the LHD is shown in Fig. 1, and the equilibrium profile of the rotational transform is shown in Fig. 2.

The same calculation conditions were used for the modified and original MIPS simulations. The uniform density $\rho=B_{\mathrm{ax}}^{2} / \mu_{0} V_{\mathrm{A}}^{2}$ was assumed, where $B_{\mathrm{ax}}$ is the equilibrium magnetic field strength on the magnetic axis and $V_{\mathrm{A}}$ is the Alfvén speed. The resistivity $\eta=10^{-6} \mu_{0} V_{\mathrm{A}} R_{\mathrm{c}}$, the viscosity $\nu=10^{-8} \mu_{0} V_{\mathrm{A}} R_{\mathrm{c}}$, and the thermal diffusion coefficient $\chi=10^{-8} \mu_{0} V_{\mathrm{A}} R_{\mathrm{c}}$ were assumed, where $R_{\mathrm{c}}$ is the major radius of the center of the calculation region. The adiabatic constant $\gamma=5 / 3$ was used. The simulation region was $2.55 \mathrm{~m}<R$ $<4.75 \mathrm{~m}, 0 \leq \phi<2 \pi$, and $-1.1 \mathrm{~m}<Z<1.1 \mathrm{~m}$; thus, $R_{\mathrm{c}}$ $=3.6 \mathrm{~m}$. The number of grid points was $(128,640$, and 128) in each direction. The time increment $\Delta t=1.6 \times 10^{-2} L / V_{\mathrm{A}}$ was used, where $L=1 \mathrm{~m}$. The same initial perturbations were randomly given by the use of the vector potential for both simulations. Here, the same random seeds were used so that the initial perturbations were identical.

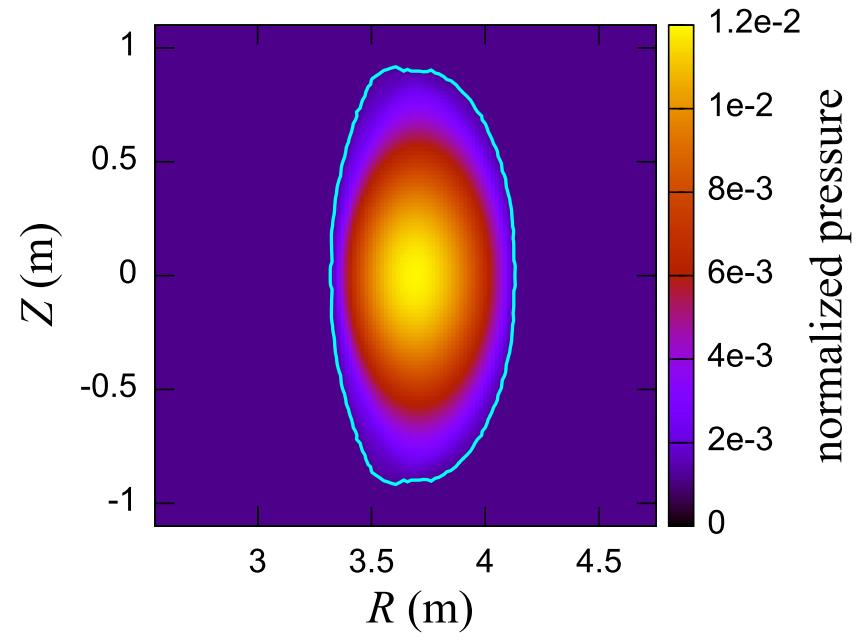

FIG. 1. Equilibrium pressure profile on the vertically elongated poloidal plane of the LHD. The light blue line indicates the plasma boundary calculated by the contour of the pressure. The pressure was normalized by $B_{\mathrm{ax}}^{2}$.

\section{COMPARISON OF MODIFIED AND ORIGINAL LINEAR MIPS SIMULATION RESULTS}

Figure 3 shows $F_{\mathrm{e}}\left(\equiv\left|\boldsymbol{B}_{0}\left(\nabla \cdot \boldsymbol{B}_{1}\right)\right| /\left|\boldsymbol{J}_{1} \times \boldsymbol{B}_{0}+\boldsymbol{J}_{0} \times \boldsymbol{B}_{1}\right|\right)$ profiles on the vertically elongated poloidal plane of the LHD at $t=1280$ in the (a) modified and (b) original linear MIPS simulations. Here, $\boldsymbol{B}_{0}\left(\nabla \cdot \boldsymbol{B}_{1}\right)$ is the error force term yielded from the $\nabla \cdot \boldsymbol{B}_{1}$ error. It is included in the Lorentz force term as follows:

$$
\boldsymbol{J} \times \boldsymbol{B}=\boldsymbol{B} \cdot \nabla \boldsymbol{B}-\frac{1}{2} \nabla B^{2}=\nabla \cdot\left[\boldsymbol{B} \boldsymbol{B}-\frac{1}{2} B^{2} \mathbf{I}\right]-\boldsymbol{B}(\nabla \cdot \boldsymbol{B}),
$$

where $\mathbf{I}$ is the unit tensor. Figure 3 shows that $\nabla \cdot \boldsymbol{B}_{1}$ in the modified version was so small that it can be regarded as zero. On the other hand, finite $\nabla \cdot \boldsymbol{B}_{1}$ was distributed inside the plasma in the original version. Figure 4 shows the time evolutions of the kinetic energy in each simulation. These were evaluated as a volume integral over the simulation region. The kinetic energy in the modified one was slightly larger than that in the original one. Table I presents growth rates $\gamma_{\mathrm{g}}$ in each simulation. Although the modified one had a

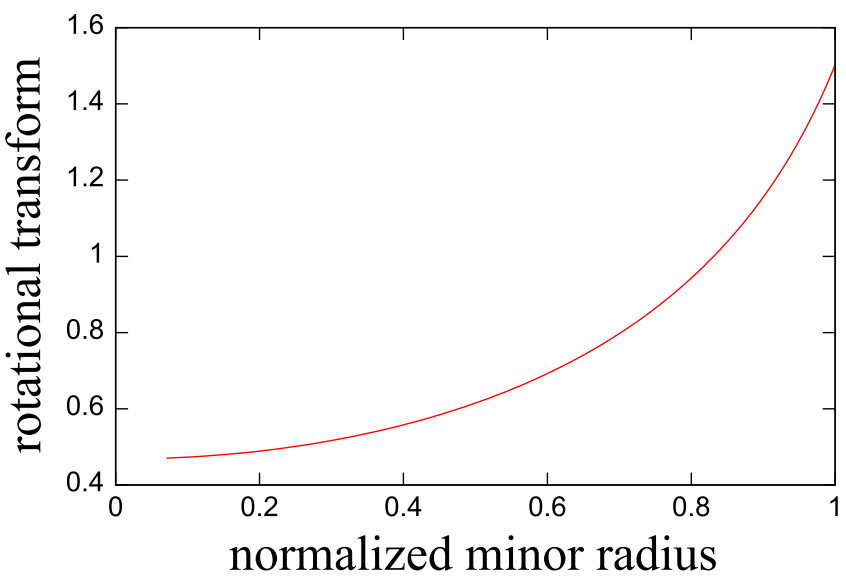

FIG. 2. Equilibrium profile of the rotational transform. 

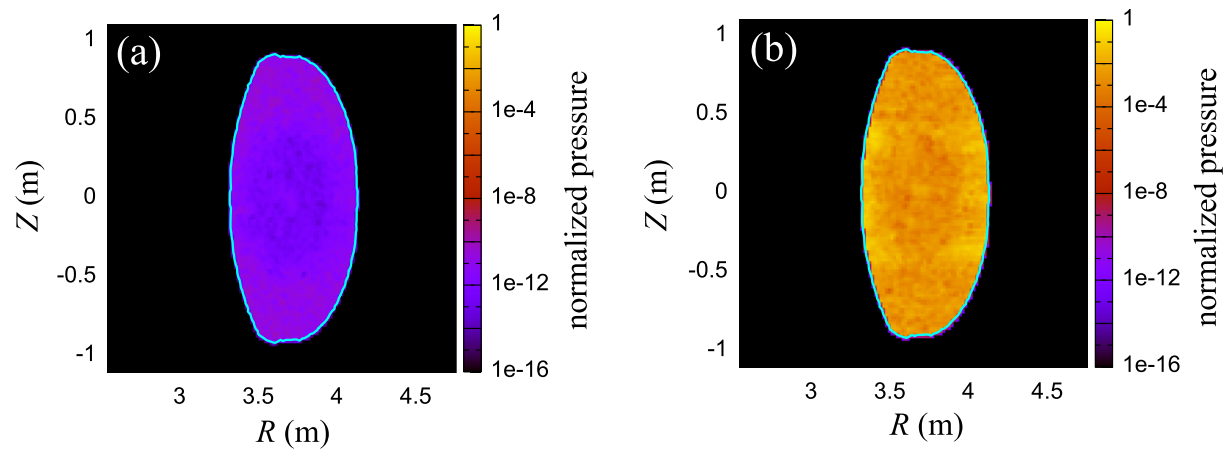

FIG. 3. $F_{\mathrm{e}}\left(\equiv\left|\boldsymbol{B}_{0}\left(\nabla \cdot \boldsymbol{B}_{1}\right)\right| / \mid \boldsymbol{J}_{1} \times \boldsymbol{B}_{0}\right.$ $\left.+\boldsymbol{J}_{0} \times \boldsymbol{B}_{1} \mid\right)$ profiles on the vertically elongated poloidal plane of the LHD at $t=1280$ in the (a) modified and (b) original linear MIPS simulations. The light blue line indicates the plasma boundary. $F_{\mathrm{e}}=0$ is given outside the plasma.

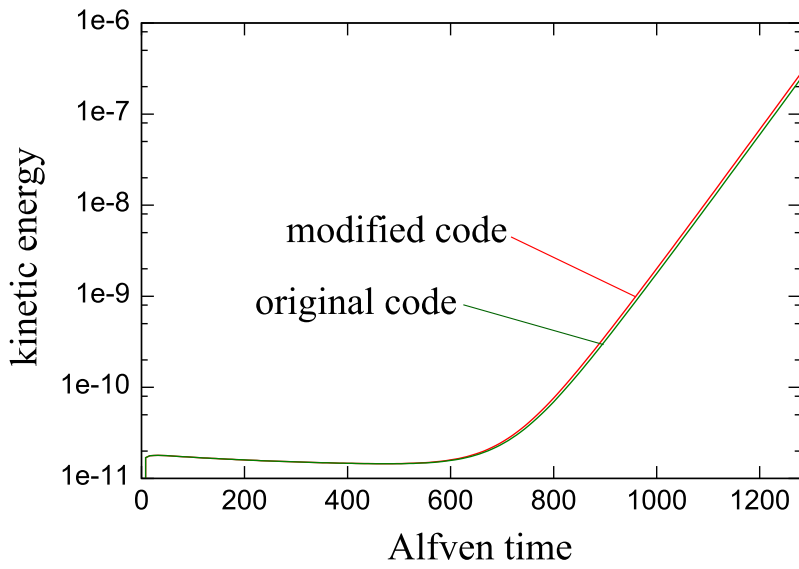

FIG. 4. Time evolutions of the kinetic energy in the simulation by the modified (red) and original (green) linear MIPS codes. These were evaluated as the volume integral over the simulation region. The kinetic energy was normalized by $B_{\mathrm{ax}}^{2} / \mu_{0}$. The Alfvén time was defined as the time normalized by $L / V_{\mathrm{A}}$.

TABLE I. Growth rates $\gamma_{\mathrm{g}}$ in the modified and original MIPS simulations. These were the half value of the slope in the semilog graph shown in Fig. 4 at $960 \leq t \leq 1280$ (from 60000 time step to 80000 ).

\begin{tabular}{lcc}
\hline \hline & Modified & Original \\
\hline$\gamma_{\mathrm{g}}$ & $8.79 \times 10^{-3}$ & $8.78 \times 10^{-3}$ \\
\hline \hline
\end{tabular}

slightly higher growth rate, the difference was negligible. Figure 5 shows the growth rates for each set of mode numbers $m / n$, where $m$ is the poloidal mode number and $n$ is the toroidal mode number. The growth rates for each mode in the modified one showed small but negligible differences.

Figure 6 shows the radial profiles of the dominant Fourier modes of the perturbed pressure for each $n$. There were a few differences between the results of the modified and original simulations. Table II presents the peak values and full widths at half maximum (FWHMs) of the Fourier modes of the perturbed pressure for $m / n=$ (a) $2 /-1$, (b) $4 /-2$, (c) $5 /-3$, (d) $6 /-3$, and (e) $7 /-4$, which were the five most dominant Fourier modes. The peak value for $m / n$ $=2 /-1$ in the modified one was almost the same as that in the original one. On the other hand, the peak values for the $m / n=4 /-2,5 /-3,6 /-3,7 /-4$ modes in the modified one were different from those in the original one. The $\nabla \cdot \boldsymbol{B}_{1}$ error shown in Fig. 3 was expected to yield these differences. Larger $m$ and $n$ reduced the peak values ratios. This means that introducing the vector potential mainly affects perturbations with a short wavelength. The FWHMs in the modified one were almost the same as those in the original one. Thus, although the mode widths are hardly affected by the $\nabla \cdot \boldsymbol{B}_{1}$ error, the peak values of the Fourier mode can be affected by the $\nabla \cdot \boldsymbol{B}_{1}$ error. Table III ranks the peak values of the dominant Fourier modes. The five most dominant Fourier modes in the modified one were the same as those in the original one.

\section{SUMMARY AND DISCUSSION}

The vector potential was introduced to the linear MIPS code, and the modified and original linear MIPS simulation results were compared. The results showed that the modified code can successfully remove the $\nabla \cdot \boldsymbol{B}_{1}$ error. The $\nabla \cdot \boldsymbol{B}_{1}$ error was so small in the modified MIPS simulation that it can be regarded as zero although finite $\nabla \cdot \boldsymbol{B}_{1}$ was distributed inside the plasma in the original one. In addition, the $\nabla \cdot \boldsymbol{B}_{1}$ error can yield incorrect results especially for analyses of the mode structure. The differences between the two simulation results were not so great, so the modified code was confirmed to work appropriately. Moreover, this would be evidence that the $\nabla \cdot \boldsymbol{B}_{1}$ error does not affect the comprehensive results for such typical linear instability analyses. In this paper, we did not refer to which code is better or closer
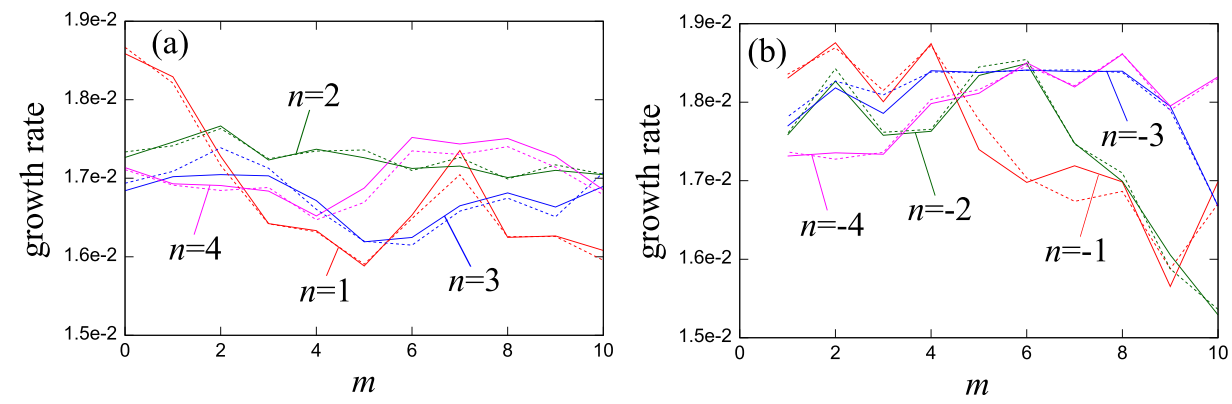

FIG. 5. Growth rates for each set of mode numbers $m / n$, where $m$ is the poloidal mode number and $n$ is the toroidal mode number: (a) positive $n$ and (b) negative $n$. The solid and dashed lines denote the resultants in the modified and original linear MIPS simulations, respectively. These were calculated by the time evolutions of Fourier modes of the kinetic energy. 


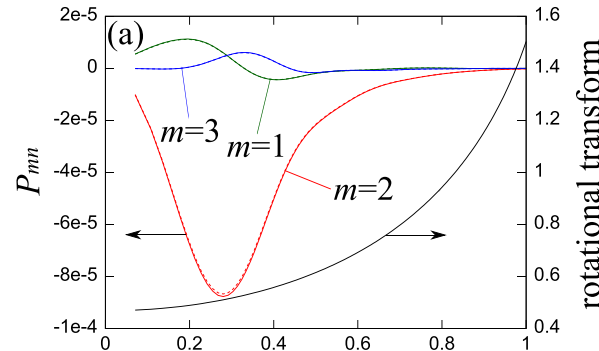

normalized minor radius

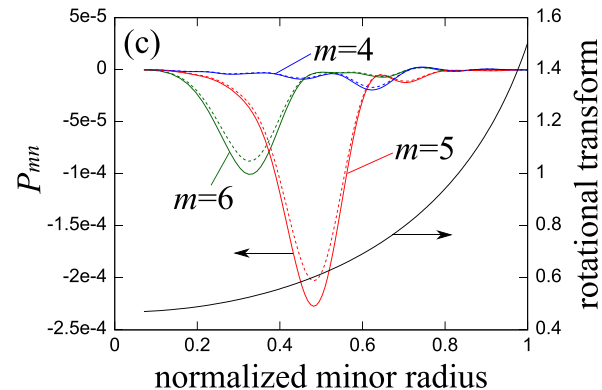

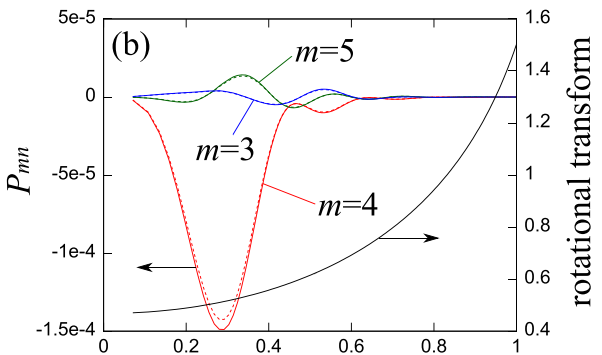

normalized minor radius

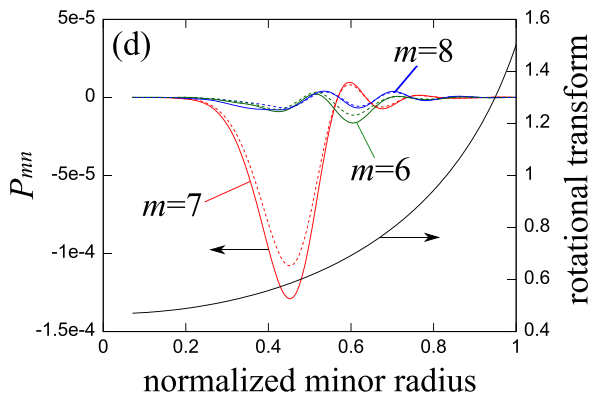

FIG. 6. Radial profiles of dominant Fourier modes for $n=$ (a) -1 , (b) -2 , (c) -3 , and (d) -4 . The solid and dashed lines denote the results in the modified and original linear MIPS simulations, respectively. $P_{m n}$ was normalized by $B_{\mathrm{ax}}^{2}$.
TABLE II. Peak values and full widths at half maximum (FWHMs) of the Fourier modes of the perturbed pressure for $m / n=$ (a) $2 /-1$, (b) $4 /-2$, (c) $5 /-3$, (d) $6 /-3$, and (e) $7 /-4 . P_{\mathrm{v}}$ and $W$ represent the peak values and FWHMs, respectively.

\begin{tabular}{lccc}
\hline \hline & Peak value $P_{\mathrm{v}}$ & Ratio $\frac{P_{\mathrm{v}}(\text { original })}{P_{\mathrm{v}}(\text { modified })}$ & FWHM $W$ \\
\hline (a) & & & \\
Modified & $-8.77 \times 10^{-5}$ & - & 0.26 \\
Original & $-8.67 \times 10^{-5}$ & $99 \%$ & 0.26 \\
(b) & & & \\
Modified & $-1.49 \times 10^{-4}$ & - & 0.18 \\
Original & $-1.42 \times 10^{-4}$ & $95 \%$ & 0.18 \\
(c) & & - & \\
Modified & $-2.27 \times 10^{-5}$ & $89 \%$ & 0.16 \\
Original & $-2.03 \times 10^{-5}$ & - & 0.16 \\
(d) & $-1.00 \times 10^{-4}$ & $88 \%$ & 0.16 \\
Modified & $-8.78 \times 10^{-5}$ & - & 0.16 \\
Original & $-1.29 \times 10^{-4}$ & $84 \%$ & 0.15 \\
(e) & $-1.08 \times 10^{-4}$ & & 0.15 \\
Modified & Original & &
\end{tabular}

TABLE III. Peak value ranking of dominant Fourier modes.

\begin{tabular}{lcc}
\hline \hline Rank & Modified & Original \\
\hline 1 & $5 /-3$ & $5 /-3$ \\
2 & $4 /-2$ & $4 /-2$ \\
3 & $7 /-4$ & $7 /-4$ \\
4 & $6 /-3$ & $6 /-3$ \\
5 & $2 /-1$ & $2 /-1$ \\
\hline \hline
\end{tabular}

to reality because there are no significant differences in two simulations.

Introducing the vector potential was found to mainly affect perturbations with a short wavelength. One reason would be that the value of $\nabla \cdot \boldsymbol{B}_{1}$ error is larger for the high spatial frequency mode. The more reliable reason is that the smoothing scheme ${ }^{9}$ is employed in the MIPS code for stable calculation. This smoothing scheme consists of the binomial and compensated filters that are applied at the same time step in succession. This scheme has a characteristic that perturbations with a short wavelength are mainly removed. ${ }^{9}$ The original code applies this scheme to $\boldsymbol{V}, \boldsymbol{B}_{1}$, and $P_{1}$ once every 100 time steps. On the other hand, the modified code applies it to $\boldsymbol{V}, \boldsymbol{A}_{1}$, and $P_{1}$ one every 100 time steps. Thus, the smoothing scheme is applied to different quantities in the original and modified codes. This smoothing scheme has a important problem that it breaks $\nabla \cdot \boldsymbol{B}_{1}=0$ in the original code because it is not considered to guarantee $\nabla \cdot \boldsymbol{B}_{1}=0$. Therefore, introducing the vector potential simultaneously enables the guarantee of $\nabla \cdot \boldsymbol{B}_{1}=0$ and the stable calculation. In addition, both the $\nabla \cdot \boldsymbol{B}_{1}$ error and the smoothing scheme might affect the high frequency mode simultaneously. We could not divide these two reasons. This problem is our future work.

The modified code would be useful for comprehensively investigating the effect of $\nabla \cdot \boldsymbol{B}_{1} \neq 0$. As stated in Section I, this code would also be useful for verifying our prediction that a large $\nabla \cdot \boldsymbol{B}_{1}$ error is expected to yield instabilities with an undesirable mode and disturb the analysis of a specified single mode instability. These issues will be investigated in future works.

\section{ACKNOWLEDGMENTS}

The authors are grateful to Professor Y. Todo and Professor M. Sato for the many useful discussions and to Professor M. Itagaki and Dr. R. Ueda for their help. Numerical analyses presented in this paper were carried out with the HITACHI SR16000 and FUJITSU FX100 plasma simulator of the National Institute for Fusion Science (NIFS). This work was partially supported by NIFS15KNXN307.

${ }^{1}$ J. U. Brackbill and D. C. Barnes, J. Comput. Phys. 35, 426 (1980).

${ }^{2}$ O. Motojima, H. Yamada, A. Komori, N. Ohyabu, K. Kawahata, O. Kaneko, S. Masuzaki, A. Ejiri, M. Emoto, H. Funaba, M. Goto, K. Ida, H. Idei, S. Inagaki, N. Inoue, S. Kado, S. Kubo, R. Kumazawa, T. Minami, J. 
Miyazawa, T. Morisaki, S. Morita, S. Murakami, S. Muto, T. Mutoh, Y. Nagayama, Y. Nakamura, H. Nakanishi, K. Narihara, K. Nishimura, N. Noda, T. Kobuchi, S. Ohdachi, Y. Oka, M. Osakabe, T. Ozaki, B. J. Peterson, A. Sagara, S. Sakakibara, R. Sakamoto, H. Sasao, M. Sasao, K. Sato, M. Sato, T. Seki, T. Shimozuma, M. Shoji, H. Suzuki, Y. Takeiri, K. Tanaka, K. Toi, T. Tokuzawa, K. Tsumori, K. Tsuzuki, I. Yamada, S. Yamaguchi, M. Yokoyama, K. Y. Watanabe, T. Watari, Y. Hamada, K. Matsuoka, K. Murai, K. Ohkubo, I. Ohtake, M. Okamoto, S. Satoh, T. Satow, S. Sudo, S. Tanahashi, K. Yamazaki, M. Fujiwara, and A. Iiyoshi, Phys. Plasmas 6, 1843 (1999).

${ }^{3}$ K. Y. Watanabe, S. Masamune, Y. Takemura, H. Funaba, S. Sakakibara, F. Watanabe, K. Tanaka, S. Ohdachi, K. Toi, Y.
Narushima, and LHD Experiment Group, Phys. Plasmas 18, 056119 (2011).

${ }^{4}$ R. Ueda, M. Sato, K. Y. Watanabe, Y. Matsumoto, Y. Suzuki, M. Itagaki, S. Oikawa, and Y. Todo, Plasma Fusion Res. 8, 2403157 (2013).

${ }^{5}$ Y. Todo, N. Nakajima, M. Sato, and H. Miura, Plasma Fusion Res. 5, S2062 (2010).

${ }^{6}$ C. R. Evans and J. F. Hawley, Astrophys. J. 332, 659 (1988).

${ }^{7}$ K. Harafuji, T. Hayashi, and T. Sato, J. Comput. Phys. 81, 169 (1989).

${ }^{8}$ Y. Suzuki, N. Nakajima, K. Y. Watanabe, Y. Nakamura, and T. Hayashi, Nucl. Fusion 46, L19 (2006).

${ }^{9}$ C. Birdsall and A. B. Langdon, Plasma Physics via Computer Simulation (McGraw-Hill, New York, 1985) p. 437. 